\title{
Effectiveness of vaccine against pandemic influenza A/H1N1 among people with underlying chronic diseases: cohort study, Denmark, 2009-10
} (c) (1) \& 8 OPEN ACCESS

\author{
Hanne-Dorthe Emborg epidemiologist, Tyra Grove Krause senior consultant, Anders Hviid senior \\ investigator, Jacob Simonsen statistician, Kåre Mølbak director, state epidemiologist
}

Division of Epidemiology, Statens Serum Institut, 2300 Copenhagen, Denmark

\begin{abstract}
Objective To determine the effectiveness of an adjuvanted monovalent vaccine against pandemic influenza $\mathrm{A} / \mathrm{H} 1 \mathrm{~N} 1$ among people with underlying chronic diseases.

Design Historical cohort study.

Setting Mandatory national reporting systems, 2 November 2009 to 31 January 2010, Denmark.

Participants 388069 people under 65 years of age with a diagnosis in the past five years of at least one underlying disease expected to increase the risk of severe illness after influenza.

Main outcome measures Laboratory confirmed $\mathrm{H} 1 \mathrm{~N} 1$ infection and influenza related hospital admission with laboratory confirmed H1N1 infection. Estimates of vaccine effectiveness were adjusted for age and underlying disease.

Results The effectiveness of pandemic vaccine against confirmed $\mathrm{H} 1 \mathrm{~N} 1$ infection 14 days after one dose of vaccine was $49 \%$ (95\% confidence interval $10 \%$ to $71 \%$ ). The effectiveness of vaccine against admission to hospital for confirmed H1N1 infection was $44 \%$ (-19\% to $73 \%)$.

Conclusions The adjuvanted monovalent vaccine against pandemic influenza A/H1N1 was offered late in the 2009-10 influenza season. Among chronically ill people, this vaccine offered protection against laboratory confirmed $\mathrm{H} 1 \mathrm{~N} 1$ infection but only offered non-significant protection against influenza related hospital admissions confirmed as $\mathrm{H} 1 \mathrm{~N} 1$ infection. This finding is of public health relevance because the population of chronically ill people is a major target group for pandemic vaccinations and because of the delayed availability of pandemic vaccines in a forthcoming pandemic.
\end{abstract}

\section{Introduction}

Early in 2009 a novel type of influenza, A/H1N1 of swine origin, emerged in Mexico and subsequently spread worldwide. As a result of this spread, the World Health Organization declared the influenza pandemic as phase 6 in June 2009. ${ }^{1}$ Several vaccines against this influenza type were developed rapidly and licensed based on approval of mock-up vaccines, as well as on limited data on immunogenicity. To obtain data on clinical effectiveness, several countries set up studies on vaccine effectiveness and in these observational studies suggested estimates for vaccine effectiveness in the general population ranged between $72 \%$ to more than $90 \% .^{2-7}$

People with underlying conditions that increase the risk of severe illness and death after influenza infection constitute a first line target group for vaccinations. However, vaccine effectiveness estimates from this risk group have been limited. ${ }^{6}{ }^{8}$ Denmark has a unique opportunity to assess vaccine effectiveness because Danish residents are each given a civil registration number, a unique personal identifier that enables linkage of medical data from several national registers. ${ }^{9}$ During the influenza pandemic, registration of pandemic vaccination was mandatory and all positive influenza test results were reported to a central database. Furthermore, all hospital admissions are registered by discharge diagnosis in the national hospitals discharge registry. These registers allow Denmark to carry out large scale register based studies on vaccine effectiveness.

We determined vaccine effectiveness of an adjuvanted monovalent vaccine among all Danish people under 65 years of age with underlying disease against clinical outcomes, including laboratory confirmed infection with $\mathrm{H} 1 \mathrm{~N} 1$ and influenza related hospital admission based on confirmed infection with $\mathrm{H} 1 \mathrm{~N} 1$.

\section{Methods}

The study was designed as a historical cohort study of people aged less than 65 years with underlying disease and covering the period 2 November 2009 to 31 January 2010, when 
vaccinations against $\mathrm{H} 1 \mathrm{~N} 1$ were offered and transmission of pandemic influenza was ongoing. The study cohort, vaccinations, and outcomes were constructed or ascertained using several data sources.

\section{National vaccination registry}

In Denmark only Pandemrix (GlaxoSmithKline), an adjuvanted monovalent vaccine, was used (3.75 $\mu$ g haemagglutinin with AS03 as adjuvant) for vaccination against pandemic influenza 2009. ${ }^{10}$ Vaccinations started on 2 November (week 45) 2009 and were mainly provided by general practitioners but also by private vaccination clinics and hospitals. It was mandatory for all vaccine providers to electronically register an individual's civil registration number and date of vaccination. In contrast, registration of the trivalent influenza vaccine for the 2009-10 season was not mandatory. Seasonal vaccine was, however, offered free to risk groups, including those with chronic illnesses. To be reimbursed for the cost of the vaccine the vaccine provider had to register the individual's civil registration number, the date of vaccination, and the vaccine given. This information was collected in a national database; it is estimated that more than $95 \%$ of all seasonal vaccines given to people with chronic diseases are registered. Registration of pandemic vaccinations was also considered to be close to complete as general practitioners, who gave $91 \%$ of the vaccinations, had to register each administered vaccine to be reimbursed for the cost of the vaccine.

\section{National influenza database}

In 2009, influenza A became a disease that had to be reported by laboratories. To ascertain the diagnosis of acute respiratory tract infection, general practitioners and hospital clinicians took swab samples from patients throughout the pandemic. Before week 46 it was recommended to take swab samples from patients with sudden onset of illness with fever, myalgia, and respiratory tract symptoms. From week 46 onwards, medical staff were advised to take swab samples only from those who were at risk of being admitted to hospital with serious influenza symptoms. If $\mathrm{H} 1 \mathrm{~N} 1$ was detected by real time polymerase chain reaction the civil registration number of the patient and date of sampling were registered in a national database. This database contains 5497 laboratory confirmed H1N1 test results from patients who had swab samples taken by general practitioners, as well as by hospitals throughout the pandemic. Most of these tests were done for clinical reasons. From week 47 onwards about $40 \%$ of tests were carried out for patients admitted to hospital with confirmed H1N1 infection.

\section{National discharge registry}

The discharge registry covers the Danish population who have been treated in a Danish hospital. The registry has tracked $99.4 \%$ of discharges from all Danish acute care, non-psychiatric hospitals since 1977 and all hospital outpatient and emergency department visits since $1995 .{ }^{11}$ This registry contains data on all hospital admissions and outpatient contacts, with diagnoses, actions, and surgical procedures of the 5.5 million Danish inhabitants. Data are coded with ICD-10 codes (international classification of diseases, 10th revision) by medical staff and entered into electronic health records. ${ }^{12}$ All Danish residents are identified by their civil registration number.

\section{Measurement of influenza-like illness}

The sentinel surveillance system is a voluntary reporting system of up to 250 general practitioners from all regions of Denmark.
Participating doctors provide the total number of consultations and age specific numbers of consultations for influenza-like illness on a weekly basis. ${ }^{13}$

\section{Registry linkage}

For all Danish residents aged less than 65 years, we used the national hospital discharge registry to construct a five year history of primary (first listed) and secondary hospital discharge diagnoses based on admissions to hospitals and visits to outpatient departments and emergency rooms. For children under 5 years, we based the history of discharge diagnoses on their lifetime history. We included in the study those people who had been admitted to hospital and diagnosed as having specific chronic diseases that were expected to increase the risk of severe illness and death after influenza (see web extra table). The cohort included 388069 people alive on 2 November 2009 and without laboratory confirmed H1N1 infection before this date. We obtained information on survival status, laboratory confirmed H1N1 infection, pandemic vaccination status, 2009-10 seasonal influenza vaccine status, and influenza related admission to hospitals by registry linkage using the civil registration number as the linking variable. The ICD-10 codes used to define potential influenza related admissions to hospitals included severe acute respiratory tract infections, acute respiratory distress syndrome, influenza, and febrile convulsions (see web extra table). Influenza related admission to hospitals confirmed as $\mathrm{H} 1 \mathrm{~N} 1$ infections were defined by a positive swab sample taken from seven days before to seven days after admission to hospital with an influenza related illness. No one had more than one laboratory confirmed H1N1 infection.

\section{Statistical analysis}

\section{Estimating the effectiveness of pandemic vaccine}

We estimated the effectiveness of pandemic vaccine using proportional hazards regression models. Outcomes included laboratory confirmed H1N1 infection and influenza related hospital admission confirmed as H1N1 infection. Pandemic vaccination status was included as a time dependent covariate; where two doses were given, we considered only the first dose. As the implementation of the vaccination programme coincided with variations in influenza transmission, we included calendar time in days as the underlying time scale. Vaccine effectiveness was estimated as (1-hazard ratio) $\times 100$. Age (years) was grouped into seven categories: 0-9, 10-19, 20-29, 30-39, 40-49, 50-59, and 60-64. The number of different chronic disease diagnoses for each individual varied from one to nine; for the present study we grouped together four or more diagnoses. Using the change in estimate method, we defined age groups and number of different chronic disease diagnoses as confounders because these variables changed vaccine effectiveness estimates by more than $10 \%$. The models therefore contained age groups, number of chronic diagnoses, seasonal vaccine status, and pandemic vaccine status. We stratified the conditional regression analyses by age group and number of chronic disease diagnoses. A non-stratified analysis was also done to obtain estimates for the different levels of age groups and number of different chronic disease diagnoses.

We carried out a subanalysis, including an interaction term between pandemic and seasonal vaccine status, to assess if the 2009-10 seasonal influenza vaccine modified the effect of the pandemic vaccine. If individuals received a dose of the pandemic vaccine 14 days before the date of a swab sample being taken, we considered them vaccinated with the pandemic vaccine. We also analysed possible protection in the time window of 1-7 
days and 8-14 days after receiving one dose of the pandemic vaccine. The study period started 2 November 2009 and ended 31 January 2010, at the time of death, or at the time a participant experienced one of the outcomes. We used restricted cubic splines to smooth the time dependent relative hazard rate. ${ }^{14}$

\section{Sensitivity analysis to estimate the impact of sampling date $v$ date of illness onset}

As the date of illness onset was not available, we used the date of sampling as a proxy. This may potentially underestimate vaccine effectiveness as it is possible to misclassify the timing of outcome in relation to day of vaccination. Therefore we went back to interviews carried out among the first cases with H1N1 infection during July 2009. In 71 interviews where the date of onset and date of sampling were available, the difference between the dates ranged from 0 to 7 days, with a mean of 2.1 days and a median of 2.0 days. The analyses were repeated using the estimated date of onset as two days before sampling instead of the date of sampling. As the time between date of onset and the date medical attention was sought may have increased during the pandemic, we carried out a sensitivity analysis to investigate differences of 3, 4, 5, and 6 days.

Data management and statistical analyses were carried out in SAS version 9.2.

\section{Results}

In the cohort of 388069 people (8.4\% of the population aged $<65$ years), the coverage of both the pandemic vaccine and the 2009-10 seasonal influenza vaccine differed between age groups, with the lowest observed in the youngest age groups, 0-39 years (table $1 \Downarrow$ ). Most of the cohort (80.8\% of 313526 people) had only one diagnosed underlying illness. Vaccine uptake increased for both vaccines with increasing number of chronic disease diagnoses (table 1). H1N1 infections were also more likely to be confirmed with increasing number of chronic disease diagnoses and were inversely associated with age (table 1).

In total, 79988 (20.6\%) people received at least one dose of pandemic vaccine, and among those, $46 \%$ received their first dose during the first week the vaccine was available. Only one dose of the pandemic vaccine was given to 47924 (59.9\%) people, whereas $32064(40.1 \%)$ people received two doses. A total of 49435 (12.7\%) people received the 2009-10 seasonal influenza vaccine. Of these, $29691(60 \%)$ received the seasonal influenza vaccine before vaccination against pandemic influenza was initiated. Among those vaccinated with the pandemic vaccine, $42294(52.9 \%)$ received only the pandemic vaccine and 37694 (47.1\%) received both vaccines. Furthermore, 11 741 (3.0\% of the cohort) people received only the seasonal vaccine, whereas $296342(76.4 \%)$ people did not receive any of the vaccines.

The pandemic peaked at the same time as the pandemic vaccines were available (fig $1 \Downarrow$ ). According to the recommendations of the European Center for Disease Control, people are considered protected 14 days after vaccination, ${ }^{15}$ which coincides with the time when the numbers of weekly cases of influenza-like illness were declining (fig 1). Most of the second pandemic vaccine doses were given in week 49 or later. Assuming the full effect of the second dose also occurs 14 days after vaccination, most people would not be fully protected until week 51 , when influenza activity was low. On this basis any enhancement of clinical response due to the second dose of vaccine could not be estimated (fig 1).

\section{Effectiveness of pandemic vaccine against laboratory confirmed H1N1 infection}

Of 799 people who tested positive for H1N1 infection in the cohort, 718 were not vaccinated with the pandemic vaccine, 49 were vaccinated 1-7 days before the date of swabbing, 18 were vaccinated 8-14 days before the date of swabbing, and 14 were vaccinated more than 14 days before the date of swabbing. Table 1 shows the distribution of the 799 people positive for H1N1 infection on the levels of explanatory variables.

An increased risk of laboratory confirmed H1N1 infection was observed during the first 1-7 days after receiving the pandemic vaccine, with vaccine effectiveness estimates of $-112 \%(95 \%$ confidence interval $-187 \%$ to $-56 \%$ ) compared with those who did not receive either the pandemic or the seasonal influenza vaccines. In the following 8-14 days, no significant effectiveness from the pandemic vaccine was observed, and effectiveness was $49 \%$ (10\% to $71 \%$ ) more than 14 days after receiving the vaccine (table $2 \Downarrow$ ). Those who received only the 2009-10 seasonal influenza vaccine had an increased risk of laboratory confirmed H1N1 infection compared with those who were not vaccinated (hazard ratio 2.31, 95\% confidence interval 1.65 to 3.23; table 2).

The highest risk of laboratory confirmed H1N1 infection was observed in the youngest age groups, with a 23-fold higher risk among those aged $0-9$ years and a 22 -fold higher risk in those aged 10-19 years compared with the 60-64 age group. For the remaining age groups, a gradual decrease in risk was observed (table $3 \Downarrow$ ). Additionally, the risk of laboratory confirmed H1N1 infection increased with increasing number of different chronic disease diagnoses. The risk of having a laboratory confirmed H1N1 infection was five times smaller if an individual had a diagnosis of only one chronic disease compared with having four or more different ones (table 3).

For those who received both the seasonal and the pandemic influenza vaccines, the combined effect of these vaccines did not differ from the effect observed among those who received only the pandemic vaccine $(\mathrm{P}=0.93)$.

Figure $2 \Downarrow$ shows the relative hazard rates of laboratory confirmed H1N1 infections over time between those who received the pandemic vaccine and those who did not receive either of the two vaccines. The relative hazard rate decreased gradually from the day of vaccination (day 0) to about day 21 , indicating that the clinical effect of the pandemic vaccine increased until about 21 days after vaccination.

\section{Sensitivity analysis}

Accounting for the estimated delay of two days between date of onset and date of swabbing did not change the effectiveness estimates of the pandemic vaccine 14 days or more after receiving the pandemic vaccine. The date of sampling was therefore kept as a proxy for disease onset in the final model. When accounting for a longer delay between date of onset and date of sampling, a few per cent decrease in vaccine effectiveness was observed.

\section{Effectiveness of pandemic vaccine against influenza related admission to hospitals confirmed as H1N1 infection}

Among the 229 patients that were admitted to hospital, 188 were not vaccinated with the pandemic vaccine, 25 were vaccinated 1-7 days before admission, eight were vaccinated 8-14 days before admission, and eight were admitted more than 14 days after vaccination. Eighty one per cent of the swab 
samples were collected on the day of or the day after admission to hospital.

An increased risk of $\mathrm{H} 1 \mathrm{~N} 1$ related admission to hospital was observed during the first 1-7 days after receiving the pandemic vaccine, with vaccine effectiveness estimates of $-258 \%(95 \%$ confidence interval $-464 \%$ to $-127 \%$ ) compared with those who did not receive either the pandemic or the seasonal vaccines. No significant effectiveness of the pandemic vaccine was observed 8-14 days after vaccination and the analysis indicated a non-significant protective effect, with $44 \%$ (95\% confidence interval $-19 \%$ to $73 \%$ ) vaccine effectiveness 14 days or more after receiving the pandemic vaccine (table 2). Those who received only the 2009-10 seasonal influenza vaccine had an increased risk of H1N1 related hospital admission (hazard ratio $2.55,95 \%$ confidence interval 1.38 to 4.70 ; table 2 ).

As found for laboratory confirmed H1N1 infection, the highest risk of admission to hospital was observed in the youngest age groups, with an 18-fold higher risk among 0-9 year olds compared with 60-64 year olds. The risk of admission decreased gradually with age from a 10-fold higher risk among 10-19 year olds to a twofold higher risk in 50-59 year olds, compared with the 60-64 year olds (table 3). Furthermore, the risk of H1N1 related admissions to hospital increased with increasing number of different chronic disease diagnoses (table 3).

For those who received both the seasonal and the pandemic vaccines the combined effect of these vaccines did not differ from the effect observed among people who received only the pandemic vaccine $(\mathrm{P}=0.82)$.

\section{Discussion}

In this national cohort study we estimated the effectiveness of the pandemic influenza $\mathrm{A} / \mathrm{H} 1 \mathrm{~N} 1$ vaccine against laboratory confirmed H1N1 infection and influenza related admissions to hospital confirmed as H1N1 infection in almost 390000 people aged less than 65 years with major underlying chronic diseases. In this target group, monovalent pandemic vaccine protected against laboratory confirmed H1N1 infection 14 days after vaccination, with $49 \%$ vaccine effectiveness, and indicated a non-significant protection against $\mathrm{H} 1 \mathrm{~N} 1$ related admissions to hospital. In contrast, during the first 1-7 days after receiving the pandemic vaccine, the risk of both outcomes was increased compared with people who received either the pandemic vaccine or the seasonal influenza vaccine. Seasonal vaccine alone did not offer any protection against the novel H1N1 infection and was associated with an increased risk of both outcomes.

\section{Comparison with other studies}

The estimates of vaccine effectiveness against verified influenza were lower in people with chronic diseases than in other studies that comprised samples from the general population..$^{2-7}$ In a Scottish study, the vaccine effectiveness for cardiovascular related conditions alone was $71.1 \%$ and if cardiovascular related conditions were combined with pneumonia and chronic obstructive pulmonary disease, the effectiveness of the vaccine decreased to $65 \% .^{6}$ These findings are in line with the present study, where an increase in number of different chronic disease diagnoses was associated with a higher chance of a confirmed H1N1 infection and H1N1 related hospital admission. In England, a case-control study including people who were eligible for vaccination because of underlying chronic disease showed $62 \%$ effectiveness of the pandemic vaccine against confirmed influenza. ${ }^{8}$ In Sweden, a large scale register based study found weekly vaccine effectiveness of between $87 \%$ and $95 \% .^{7}$ This study was based on the general population, although significantly more vaccine failures were observed among those with chronic conditions. In the present study, which included people with often severe underlying chronic diseases, a vaccine effectiveness estimate of $49 \%$ against laboratory confirmed $\mathrm{H} 1 \mathrm{~N} 1$ infection indicated that the pandemic vaccine did protect against a relevant clinical outcome but, as expected, effectiveness was lower than in the general population. Early studies from the pandemic indicated that children and younger adults in particular were affected by $\mathrm{H} 1 \mathrm{~N} 1$ infection, similar to the findings in this study where people aged less than 20 years had the highest risk of both outcomes. ${ }^{16} 17$

A study from Canada reported an increased risk of medically attended H1N1 illness after vaccination with 2008-9 seasonal influenza vaccine..$^{18}$ Along this line, we found an increased risk of laboratory confirmed H1N1 infection and hospital admissions after vaccination with the 2009-10 seasonal influenza vaccine independent of the pandemic vaccine. In contrast, an Australian study found no evidence for either protective or increased risk of laboratory confirmed infection with $\mathrm{H} 1 \mathrm{~N} 1$ after vaccination against seasonal influenza. ${ }^{19}$ The Australian study was based on sentinel patients from general practitioners, whereas our study was based on people with often severe chronic illness. Although we adjusted for several different discharge diagnoses, the study population had underlying conditions and consequently was more likely to seek care and be examined, but also to get vaccinated. In addition, this population fulfilled the recommendations for swabbing. This might explain the increased risk of an influenza diagnosis in the first 1-7 days after pandemic vaccination or after seasonal vaccination. ${ }^{20}$ Hence, both the increased risk of having a diagnosis of H1N1 infection in the first week after pandemic vaccination and the independent increased risk of $\mathrm{H} 1 \mathrm{~N} 1$ infection after seasonal vaccination may be due to the design of the study and may lead to an underestimation of the effectiveness of pandemic vaccine. In addition, those who were vaccinated against pandemic or seasonal influenza had a higher risk of being exposed to influenza from other patients in the waiting room. As an alternative explanation for the increased risk of $\mathrm{H} 1 \mathrm{~N} 1$ infection after the seasonal vaccine offered in 2009-10, one group of researchers suggested that repeated immunisations effectively block the robust, complex, and cross protective immunity afforded by previous infection. This might be a possible explanation for the Canadian findings and also a possible explanation for the finding in the present $\mathrm{study}^{18}$; in particular this may be the case because the cohort consisted of people with often severe chronic conditions for whom seasonal influenza vaccine is recommended each year.

\section{Strengths and limitations of the study}

This study has several advantages. The population included the main target group for vaccination and comprised almost 390 000 people and 800 cases with laboratory confirmed influenza. This allowed us to do multivariable analysis to adjust for other variables. The Cox regression analysis allowed us in an optimal way to control for calendar time, which was an important confounder owing to the coincidence between distribution of vaccines and the decrease in transmission of $\mathrm{H} 1 \mathrm{~N} 1$ over time (fig 1). Without controlling for calendar time, vaccine effectiveness would have been overestimated. Vaccination status and occurrence of chronic diseases were registered in population based registries before the pandemic and were independent of the outcomes analysed. Influenza was defined by laboratory confirmed infection and not symptoms of influenza-like illness. Furthermore, although the vaccine registry has not been formally validated, the completeness is high because the vaccine 
providers can only recover the cost of the vaccine if they register the vaccination in the national registers. In addition, the use of complete national registers overcomes the problem of selection bias introduced by non-random missing data, which often provide problems for epidemiological studies based on active data collection.

The present study is subject to limitations. Similar to other studies in Europe on the effectiveness of the pandemic vaccine, the present study was an observational one. Neither vaccines nor swabs were given or taken at random. It is likely that knowledge of vaccination status could be an incentive for both taking or not taking a sample. However, we know from surveillance by our intensive care units that the clinicians usually did not have any knowledge or record of vaccination status in the patients' files. ${ }^{21}$ For obtaining those data the surveillance epidemiologists had to collect the information from the national registers used for the present study rather than from clinical records. We therefore consider that the clinicians, the case management, and the sample taking were independent of vaccination status.

\section{Conclusions}

The pandemic vaccine came late in the pandemic wave of autumn 2009. Among chronically ill people this vaccine offered protection against laboratory confirmed $\mathrm{H} 1 \mathrm{~N} 1$ infection, but it only offered non-significant protection against hospital admissions confirmed as H1N1 infection. These results are of public health relevance because those with underlying conditions are a major target group for influenza vaccinations, and because pandemic vaccines are likely to arrive late in forthcoming pandemics. The increased risk of confirmed H1N1 infection and hospital admissions confirmed as H1N1 infection observed after seasonal influenza vaccine might be related to health seeking behaviour among those with severe chronic diseases or lack of cross protective immunity after repeated immunisations.

Contributors: KM and TGK designed the study. H-DE, KM, and TGK drafted the manuscript. H-DE managed and analysed the data. JS helped analyse the data. AH contributed to data analysis, interpretation of the results, and writing of the manuscript. All authors reviewed and approved the final manuscript. H-DE is the guarantor.

Funding: This study was supported by European Vaccine Manufacture through a grant managed by EpiConcept. The funding source did not influence or participate in the design and conduct of the study; the collection, management, analysis, and interpretation of the data; writing the manuscript; or the decision to submit the article for publication. The researchers were independent of the funder.

Competing interests: All authors have completed the ICMJE uniform disclosure form at www.icmje.org/coi_disclosure.pdf (available on request from the corresponding author) and declare: support from European Vaccine Manufacture through a grant managed by EpiConcept; no financial relationships with any organisations that might have an interest in the submitted work in the previous three years; no other relationships or activities that could appear to have influenced the submitted work
Ethical approval: Not required.

Data sharing: No additional data available.

1 Chan M. World now at the start of 2009 influenza pandemic. 2009. www.who.int/ mediacentre/news/statements/2009/h1n1_pandemic_phase6_20090611/en/index.html.

2 Hardelid P, Fleming D, McMenamin J, Andrews N, Robertson C, Sebastianpillai P, et al. Effectiveness of pandemic and seasonal influenza vaccine in preventing pandemic influenza A(H1N1)2009 infection in England and Scotland 2009-2010. Euro Surveill 2011;16:pii,19763.

3 Valenciano M, Kissling E, Cohen J-M, Oroszi B, Barret A-S, Rizzo C, et al. Estimates of pandemic influenza vaccine effectiveness in Europe, 2009-2010: results of Influenza Monitoring Vaccine Effectiveness in Europe (I-MOVE) Multicentre Case-Control Study. PLoS Med 2011;8:e1000388.

4 Puig-Barberà J, Arnedo-Pena A, Pardo-Serrano F, Tirado-Balaguer MD, Pérez-Vilar S, Silvestre-Silvestre E, et al. Effectiveness of seasonal 2008-2009, 2009-2010 and pandemic vaccines, to prevent influenza hospitalizations during the autumn 2009 influenza pandemic wave in Castellón, Spain. A test-negative, hospital-based, case-control study. Vaccine 2010;28:7460-7.

5 Wichmann O, Stocker P, Poggensee G, Altmann D, Walter D, Hellenbrand W, et al. Pandemic influenza $A(H 1 N 1) 2009$ breakthrough infections and estimates of vaccine effectiveness in Germany 2009-2010. Euro Surveill 2010;15:pii,19561.

6 Simpson CR, Ritchie LD, Robertson C, Sheikh A, McMenamin J. Vaccine effectiveness in pandemic influenza - primary care reporting (VIPER): an observational study to assess the effectiveness of the pandemic influenza A ( $\mathrm{H} 1 \mathrm{~N} 1) \mathrm{v}$ vaccine. Health Technol Assess 2010;14:313-46.

7 Ortqvist A, Berggren I, Insulander M, de Jong B, Svenungsson B. Effectiveness of an adjuvanted monovalent vaccine against the 2009 pandemic strain of influenza $A(H 1 N 1) v$, in Stockholm County, Sweden. Clin Infect Dis 2011;52:1203-11.

8 Andrews N, Waight P, Yung C-F, Miller E. Age-specific effectiveness of an oil-in-water adjuvanted pandemic (H1N1) 2009 vaccine against confirmed infection in high risk groups in England. J Infect Dis 2011;203:32-9.

9 Pedersen CB, Gøtzsche H, Møller JO, Mortensen PB. The Danish civil registration system. A cohort of eight million persons. Dan Med Bull 2006;53:441-9.

10 Johansen K, Nicoll A, Ciancio BC, Kramarz P. Pandemic influenza A(H1N1) 2009 vaccines in the European Union. Euro Surveill 2009;14:19361.

11 Andersen TF, Madsen M, Jørgensen J, Mellemkjoer L, Olsen JH. The Danish National Hospital Register. A valuable source of data for modern health sciences. Dan Med Bull 1999;46:263-8.

12 World Health Organization. International classification of diseases (ICD). 2011. www.who. int/classifications/icd/en/.

13 Harder KM, Andersen PH, Bæhr I, Nielsen LP, Ethelberg S, Glismann S, et al. Electronic real-time surveillance for influenza-like illness: experience from the 2009 influenza $A(H 1 N 1)$ pandemic in Denmark. Euro Surveill 2011;16:pii,19767.

14 Harrell FE. Regression modelling strategies: with applications to linear models, logistic regression, and survival analysis. New Springer, 2001.

15 ECDC. Protocol for case-control studies to measure pandemic and seasonal influenza vaccine effectiveness in the European Union and European Economic Area Member States. 2011. http://ecdc.europa.eu/en/publications/Publications/0907_TED_Influenza AH1N1_Measuring_Influenza_Vaccine_Effectiveness_Protocol_Case_Control_Studies. pdf.

16 Fraser C, Donnelly CA, Cauchemez S, Hanage WP, Van Kerkhove MD, Hollingsworth TD, et al. Pandemic potential of a strain of influenza A (H1N1): early findings. Science 2009;324:1557-61.

17 Dawood FS, Jain S, Finelli L, Shaw MW, Lindstrom S, Garten RJ, et al. Emergence of a novel swine-origin influenza A (H1N1) virus in humans. N Engl J Med 2009;360:2605-15.

18 Skowronski DM, De Serres G, Crowcroft NS, Janjua NZ, Boulianne N, Hottes TS, et al. Association between the 2008-09 seasonal influenza vaccine and pandemic $\mathrm{H} 1 \mathrm{~N} 1$ illness during spring-summer 2009: four observational studies from Canada. PLoS Med 2010;7:e1000258.

19 Kelly HA, Grant KA, Fielding JE, Carville KS, Looker CO, Tran T, et al. Pandemic influenza H1N1 2009 infection in Victoria, Australia: no evidence for harm or benefit following receip of seasonal influenza vaccine in 2009. Vaccine 2011;29:6419-26.

20 Hak E, Verheij TJM, Grobbee DE, Nichol KL, Hoes AW. Confounding by indication in non-experimental evaluation of vaccine effectiveness: the example of prevention of influenza complications. J Epidemiol Community Health 2002;56:951-5.

21 Gubbels S, Perner A, Valentiner-Branth P, Molbak K. National surveillance of pandemic influenza $A(H 1 N 1)$ infection-related admissions to intensive care units during the 2009-10 winter peak in Denmark: two complementary approaches. Euro Surveill 2010;15:pii,19743.

Accepted: 5 October 2011

\section{Cite this as: BMJ 2012;344:d7901}

This is an open-access article distributed under the terms of the Creative Commons Attribution Non-commercial License, which permits use, distribution, and reproduction in any medium, provided the original work is properly cited, the use is non commercial and is otherwise in compliance with the license. See: http://creativecommons.org/licenses/by$\mathrm{nc} / 2.0 /$ and http://creativecommons.org/licenses/by-nc/2.0/legalcode. 


\section{What is already known on this topic}

Epidemiological studies indicate that monovalent vaccine against pandemic influenza $\mathrm{A} / \mathrm{H} 1 \mathrm{~N} 1$ is more than $70 \%$ effective in the general population

Few studies have estimated the effectiveness of the pandemic vaccine in those with underlying diseases at risk of complications after influenza

Estimates of effectiveness of the 2009-10 seasonal influenza vaccine against pandemic H1N1 infection has varied from a protective effect to an increased risk of pandemic 2009 influenza

\section{What this study adds}

Monovalent pandemic vaccine offered protection against laboratory confirmed H1N1 infection among those with underlying chronic diseases, despite its late availability in the 2009 influenza pandemic

Analyses of the interaction between pandemic and seasonal vaccine status indicated that seasonal vaccine status did not impact on the effect of the pandemic vaccine

\section{Tables}

Table 1| Distribution of laboratory confirmed cases of pandemic influenza A/H1N1 and influenza related admissions to hospitals confirmed as H1N1 infection on outcome and explanatory variables in a cohort of 388<thin $>069$ people with underlying chronic disease, Denmark

\begin{tabular}{|c|c|c|c|c|c|c|c|c|c|c|c|}
\hline \multirow[b]{3}{*}{ Variables } & \multirow[b]{3}{*}{$\begin{array}{c}\text { No of } \\
\text { participants }\end{array}$} & \multirow{3}{*}{$\begin{array}{l}\% \\
\text { vaccinated } \\
\text { against } \\
\text { H1N1 }\end{array}$} & \multirow{3}{*}{$\begin{array}{l}\% \\
\text { vaccinated } \\
\text { with } \\
\text { seasonal } \\
\text { vaccine }^{*}\end{array}$} & \multirow{2}{*}{\multicolumn{4}{|c|}{$\begin{array}{c}\text { Laboratory confirmed H1N1 infection } \\
\text { Timing of pandemic vaccination }\end{array}$}} & \multirow{2}{*}{\multicolumn{4}{|c|}{$\begin{array}{c}\text { Influenza related hospital admission confirmed } \\
\text { as H1N1 infection } \\
\text { Timing of pandemic vaccination }\end{array}$}} \\
\hline & & & & & & & & & & & \\
\hline & & & & $>14$ days & 8-14 days & 1-7 days & $\begin{array}{c}\text { Not } \\
\text { vaccinated }\end{array}$ & $>14$ days & 8-14 days & $1-7$ days & $\begin{array}{c}\text { Not } \\
\text { vaccinated }\end{array}$ \\
\hline \multicolumn{12}{|l|}{$\begin{array}{l}\text { Age groups } \\
\text { (years): }\end{array}$} \\
\hline $0-9$ & 42998 & 11.3 & 1.3 & 2 & 8 & 9 & 259 & 0 & 4 & 6 & 61 \\
\hline $10-19$ & 29072 & 14.3 & 2.6 & 2 & 3 & 10 & 159 & 0 & 1 & 2 & 24 \\
\hline $20-29$ & 32681 & 11.2 & 3.2 & 1 & 3 & 3 & 51 & 1 & 2 & 2 & 19 \\
\hline $30-39$ & 62471 & 13.2 & 4.6 & 1 & 1 & 8 & 88 & 1 & 1 & 3 & 25 \\
\hline $40-49$ & 69641 & 20.0 & 11.1 & 4 & 1 & 7 & 79 & 3 & 0 & 4 & 35 \\
\hline $50-59$ & 88651 & 27.7 & 20.3 & 2 & 0 & 8 & 62 & 2 & 0 & 5 & 18 \\
\hline $60-64$ & 62555 & 32.9 & 29.5 & 2 & 2 & 4 & 20 & 1 & 0 & 3 & 6 \\
\hline
\end{tabular}

No of different

chronic

disease

diagnoses:

\begin{tabular}{lccccccccccc}
\hline 1 & 313525 & 16.6 & 9.3 & 9 & 12 & 31 & 565 & 7 & 5 & 16 & 142 \\
\hline 2 & 56062 & 33.4 & 23.4 & 2 & 6 & 12 & 105 & 0 & 3 & 4 & 28 \\
\hline 3 & 13112 & 47.2 & 35.5 & 3 & 0 & 3 & 32 & 1 & 0 & 2 & 12 \\
\hline$\geq 4$ & 5370 & 58.0 & 46.6 & 0 & 0 & 3 & 16 & 0 & 0 & 3 & 6 \\
\hline
\end{tabular}

*2009-10 seasonal influenza vaccine. 
Table 2| Estimated hazard ratios between levels of vaccine status for outcomes of laboratory confirmed pandemic influenza A/H1N1 2009 infection and influenza related admissions to hospital confirmed as H1N1 infection. Estimates obtained using proportional hazard regression with stratification on age groups and number of different chronic disease diagnoses

Hazard ratio $(95 \% \mathrm{Cl})$

Models

Laboratory confirmed H1N1 infection Influenza related hospital admission confirmed as H1N1 infection

Pandemic vaccine:

\begin{tabular}{lcccc}
\hline$>14$ days after vaccination* & 0.51 & $(0.29$ to 0.90$)$ & 0.56 & $(0.27$ to 1.19$)$ \\
\hline 8-14 days after vaccination & 1.22 & $(0.75$ to 1.99$)$ & 1.24 & $(0.60$ to 2.59$)$ \\
\hline 1-7 days after vaccination* & 2.12 & $(1.56$ to 2.87$)$ & 3.58 & $(2.27$ to 5.64$)$ \\
\hline Seasonal vaccine only & 2.31 & $(1.65$ to 3.23$)$ & 2.55 & $(1.38$ to 4.70$)$ \\
\hline No vaccine (reference) & 1 & - & 1 & - \\
\hline
\end{tabular}

${ }^{*}$ Regardless of seasonal vaccine. 
Table 3| Estimated hazard ratios between age groups and number of different chronic disease diagnoses for outcomes laboratory confirmed pandemic influenza A/H1N1 infection and influenza related admissions to hospitals confirmed as H1N1 infection. Estimates were obtained using proportional hazard regression. Models also contained pandemic and seasonal vaccine status; data on last two variables are not shown

Hazard ratio $(95 \% \mathrm{Cl})$

Model variables

Laboratory confirmed H1N1 infection Influenza related hospital admission confirmed as H1N1 infection

Age groups (years):

\begin{tabular}{|c|c|c|c|c|}
\hline $0-9$ & 23.21 & (15.49 to 34.79$)$ & 17.49 & (8.78 to 34.86$)$ \\
\hline $10-19$ & 21.79 & (14.39 to 33.00$)$ & 9.92 & (4.68 to 21.04$)$ \\
\hline $20-29$ & 6.29 & (3.96 to 10.01 ) & 7.70 & (3.60 to 16.49$)$ \\
\hline $30-39$ & 5.28 & (3.43 to 8.12$)$ & 4.76 & (2.28 to 9.92$)$ \\
\hline $40-49$ & 3.80 & (2.48 to 5.83$)$ & 5.07 & (2.53 to 10.20$)$ \\
\hline $50-59$ & 2.04 & (1.32 to 3.15$)$ & 2.01 & 0.96 to 4.19$)$ \\
\hline $60-64$ & 1 & - & 1 & - \\
\hline \multicolumn{5}{|c|}{ No of different chronic disease diagnoses: } \\
\hline 1 & 0.22 & $(0.14$ to 0.35$)$ & 0.17 & (0.083 to 0.34$)$ \\
\hline 2 & 0.40 & (0.25 to 0.66$)$ & 0.27 & $(0.13$ to 0.57$)$ \\
\hline 3 & 0.67 & (0.39 to 1.16$)$ & 0.59 & $(0.26$ to 1.34$)$ \\
\hline$\geq 4$ & 1 & - & 1 & - \\
\hline
\end{tabular}




\section{Figures}

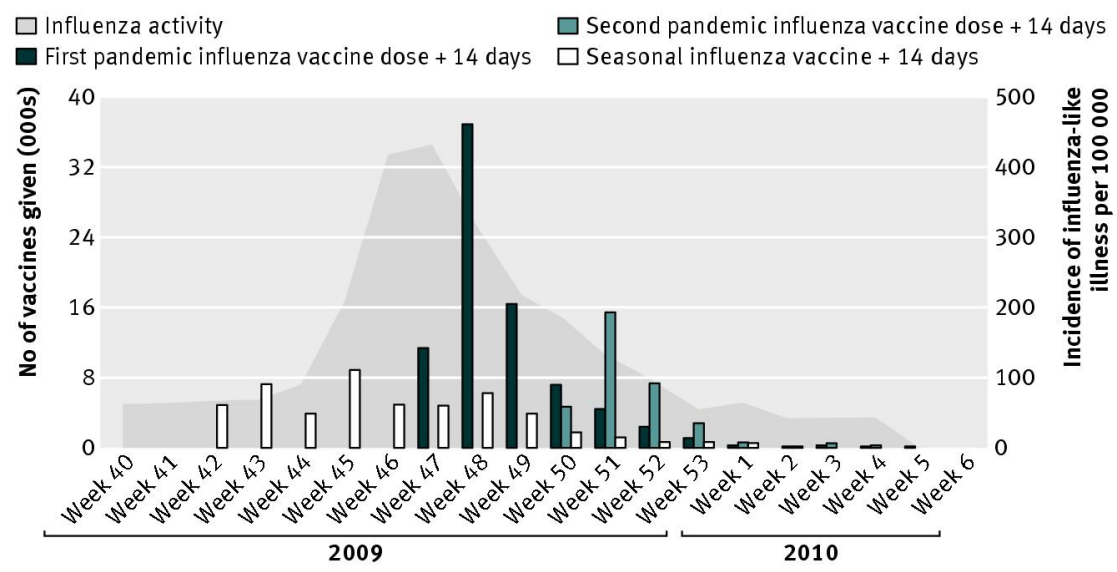

Fig 1 Weekly incidence of influenza-like illness recorded by Danish sentinel surveillance system, and number of seasonal influenza vaccines and pandemic vaccines given each week plus 14 days to people in chronic illness cohort. Bars indicating weekly vaccinations were shifted two weeks to right to indicate onset of protection

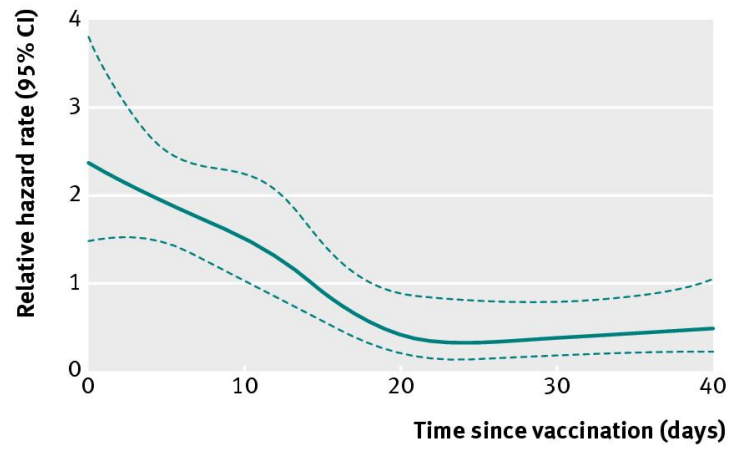

Fig 2 Relative hazard rate of laboratory confirmed $\mathrm{H} 1 \mathrm{~N} 1$ infection after vaccination with pandemic vaccine compared with people who did not receive the pandemic or seasonal influenza vaccines, Denmark 